\title{
Hardware Trojan Horses in Cryptographic IP Cores
}

\author{
Shivam BHASIN ${ }^{1}$, Jean-Luc DANGER ${ }^{1,2}$, Sylvain GUILLEY ${ }^{1,2}$, Xuan Thuy NGO $^{1, \dagger}$ and Laurent SAUVAGE $^{1,2}$ \\ ${ }^{1}$ Institut MINES-TELECOM, TELECOM-ParisTech \\ CNRS LTCI (UMR 5141), \\ 46 rue Barrault, 75634 Paris Cedex 13, France. \\ Email: firstname.lastname@telecom-paristech.fr \\ ${ }^{2}$ Secure-IC S.A.S. \\ 80 avenue des Buttes de Coësmes, 35700 Rennes, France. \\ Email: firstname.lastname@secure-ic.com \\ ${ }^{\dagger}$ Corresponding author.
}

\begin{abstract}
Detecting hardware trojans is a difficult task in general. In this article we study hardware trojan horses insertion and detection in cryptographic intellectual property (IP) blocks. The context is that of a fabless design house that sells IP blocks as GDSII hard macros, and wants to check that final products have not been infected by trojans during the foundry stage. First, we show the efficiency of a medium cost hardware trojans detection method if the placement or the routing have been redone by the foundry. It consists in the comparison between optical microscopic pictures of the silicon product and the original view from a GDSII layout database reader. Second, we analyze the ability of an attacker to introduce a hardware trojan horse without changing neither the placement nor the routing of the cryptographic IP logic. On the example of an AES engine, we show that if the placement density is beyond $80 \%$, the insertion is basically impossible. Therefore, this settles a simple design guidance to avoid trojan horses insertion in cryptographic IP blocks: have the design be compact enough, so that any functionally discreet trojan necessarily requires a complete re-place and re-route, which is detected by mere optical imaging (and not complete chip reverse-engineering).
\end{abstract}

Index Terms-Hardware trojan horses (HTH), HTH detection and insertion, optical pictures versus GDSII comparison technique, ECO place-and-route, core utilization rate (CUR).

\section{INTRODUCTION}

The semiconductor industry has spread across borders in the time of globalization. Different design phases of an Integrated Circuit (IC) may be performed at geographically dispersed locations. This coupled with the outsourcing design and fabrication to increase profitability has become a common trend in the semiconductors industry. However, this business model comes with an ample scope of introducing malicious behavior to a part of the IC. The adversary has enough scope to tamper the supply chain by maliciously implanting extra logic as Hardware Trojan Horse (HTH) circuitry into an IC [41]. This raises serious concerns about security and trustworthiness of imported products employed in critical applications like military, health, transportation, etc. HTH can be introduced in an IC at several points right from the Register Transfer Level (RTL) source code to lithographic masks fabrication. An

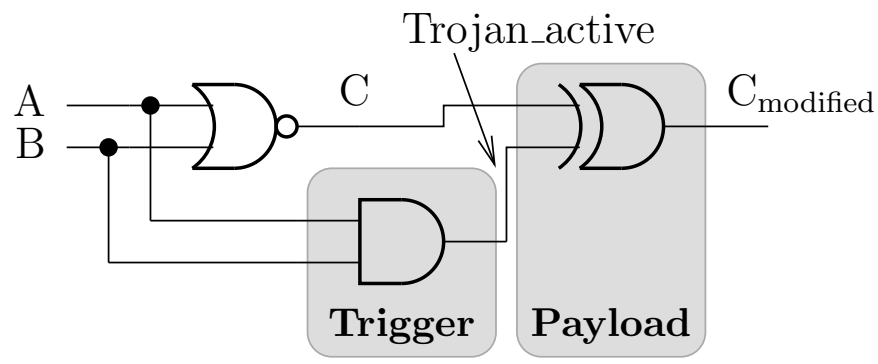

Figure 1. Minimalist Hardware Trojan Horse example

attacker can change a design netlist or subvert the fabrication process by manipulating design masks, without affecting the main functionality of the design [2].

Any HTH is composed of two main components [37]:

- Trigger: is the part of HTH used to activate the malicious activity,

- Payload: is the part of HTH used to realize/execute the malicious activity.

Fig. 1 gives an example of one simplistic HTH. In this archetype $\mathrm{HTH}$, the trigger is a simple AND gate: it tests the equality of the inputs $\mathrm{A}$ and $\mathrm{B}$ to 1 ; the payload is an XOR gate: it inverts the intermediate net $\mathrm{C}$ if the trigger is active.

An adversary can introduce a HTH which might be designed to disable or destroy a system at some future time, or to leak confidential information such as secret keys covertly to an adversary [36] by putting them to output channels. In [35], the author demonstrates an attack on a (purported militarygrade) chip using a malicious backdoor. The backdoor allows the attacker to disable all the security of the chip, reprogram cryptographic part, access secret keys, modify low-level silicon features, access unencrypted configuration bitstream or permanently damage the device. Thus protection against HTH is an open problem and an active research topic.

HTH detection is an extremely challenging problem; traditional structural and functional tests do not seem to be effective 
in targeting and detecting HTHs. Since HTH can be introduced during different design phases, the nature of HTH differs from one design phase to the others. Therefore it is difficult to find a unique detection technique for all HTH. For instance Automatic Test Pattern Generation (ATPG) methods which are used in manufacturing test for detecting defects, generally operate on the netlist of the HTH-free circuit. Existing ATPG algorithms cannot target HTH activation/detection directly [42] because HTH are designed such that they are silent most of their lifetime and have very small size relative to their host design, with featuring limited contribution into design characteristics. Such HTHs are most likely connected to nets with low controllability and/or observability [42], [5].

The state of the art principle in detection strategies of HTH can be widely classified in two categories, viz. invasive and non-invasive [26].

Invasive methods try to (prophylactically) modify the design of IC to prevent the HTH or to assist another detection technique. Chakraborty et al. have proposed a design that aims to expose the presence of a HTH in a multi-module design [11]. Salmani et al. propose a procedure to insert dummy flip-flops into logic to increase HTH activity, making the detection easier using side-channel techniques [33]. Other researchers also suggest logic additions that will make it easier to detect a HTH utilising side-channel analysis, e.g., [25] add extra logic for characterising delay times within an IC.

Non-invasive HTH detection is done by comparing the performance characteristics of an IC with a known good copy also known as the "golden circuit". Detecting HTH in a noninvasive manner can be done either at runtime or in the testing phase. The runtime detection mechanisms is combined with the countermeasures, as once a HTH is detected at runtime, attempts are made to try and continue operating by bypassing the HTH. For runtime, Bloom et al. detail a HTH detection approach that uses both hardware and software to detect two types of HTH which are DoS (Denial of Service) and combined hardware and software HTH [8]. Abramovici and Bradley added reconfigurable DEsign-For-ENabling-SEcurity (DEFENSE) logic to the functional design to implement realtime security monitors [1]. McIntyre et al. detect the presence of HTHs by executing functionally equivalent processes on multiple hardware processing elements [27]. The testing phase detection methods attempt to enhance traditional IC testing, or use side-channel analysis. For logic testing, Jha and Jha present a randomisation-based technique which probabilistically compares the functionality of the design of the circuit with the implemented circuit [19]. Chakraborty et al. suggest to test rare occurrences on an IC rather than testing for correctness [12]. The tester determines rare states that can occur within a circuit module. For side-channel analysis, Agrawal et al. present a type of detection mechanism [3]. Some known good copies of the IC are obtained and fingerprinted using one or more side-channel parameters. Other chips can then be tested against these fingerprints like path delay in [20]. Power supply transient signal analysis is used as the side channel by Rad et al. [32]. They aim at determining the smallest HTH that they can find using this technique, which can be as low as three additional gates. Banga and Hsiao [5] propose the "sustained vector technique" that is able to magnify the sidechannel differences (based on power draw) between circuits infected with HTHs and those that are not.

Most of the abovementioned techniques are indirect detection methods (e.g. reviewed in Chapter 15 of [37]). Such methods observe the effect of the HTH on the timing, the side-channel emissions, etc. The presented indirect detection methods are, under ideal circumstances, able to detect a HTH even of small size. However, countermeasures protecting cryptographic implementations often rely on perturbing the power consumption by methods like random clock jitter or masking of sensitive data, etc. Therefore, the indirect method, though still feasible, will loose efficiency in such applications. Another requirement for the indirect method is the golden reference, which can be sometimes expensive to obtain. Also side-channel based techniques are often becoming less efficient because of factors like environmental/measurement noise, process variation, or algorithmic noise. Thus HTH detection may require a huge amount of traces. On the contrary, our direct observation technique is not affected by the randomization or variations brought by countermeasures.

In this paper, we develop a new HTH detection method at the layout level of integrated circuits by using a low-cost direct visual detection. We address the scenario where the HTH is inserted by an untrusted off-shore foundry just before the fabrication of a unit. We place ourselves in the role of a designer who sends a HTH-free layout (e.g. in GDSII format - Graphic Database System version II, format of Cadence Design Systems) to a third-party foundry for fabrication but is threatened by introduction of a HTH in the GDSII by the untrusted fab. Of course an untrusted founder introducing a HTH will not share the HTH-infected GDSII with the designer. To address this issue we propose a detection technique based on image processing/comparison techniques. These techniques are used to compare the final HTH-free layout with the highquality optical snapshots of the IC. We illustrate our technique on secret key block ciphers (modified to allow for a distant fault injection); but actually it can also allow to detect the modification made in the netlist of arithmetic operations, which can lead to "bug attacks" [6] in asymmetric cryptography. Several works on insertion of HTH in cryptographic applications have been previously studied [16], [4].

This paper describes a HTH based on a backdoor that can activate a bitflip compliant with a (remote) differential fault attack. It also discusses a visual or correlation based technique capable of detecting modifications in the layout (that can somehow be considered permanent faults).

The rest of this paper is organized as follows. Section II gives a general background on AES and how fault attacks can reveal its secret key. Also in this section, a small gate count HTH is given whose principle is to trigger an artificial fault injection. Section III presents the central contribution of the paper: it explains how a visual comparison between the chip and the original GDSII can reveal HTH insertions. An 
automated method for the HTH detection based on a similarity tool (namely the cross-correlation) is then presented. Based on this detection method, a case study of HTH and results are shown in Section IV; it is applied successfully to real images and layouts. Section V gives some recommendations for the designer to implement a cryptographic IP module that would resist to HTH insertion; that is either the HTH fails to be inserted by lack of resources, or it will be detected after fabrication with our low-cost visual detection based on layout/picture comparison. Finally conclusions and perspectives are drawn in Section VI. In the appendix A, the setup to test the proposed fault-based HTH is presented.

\section{Proposal for a Low Gate Count Hardware Trojan Horse IN AES}

\section{A. General Background on Differential Fault Analysis and} AES

Physical attacks which target the implementation of cryptographic circuits (in smartcards, pay-TV and SIM cards, etc.) have been known for some years now. They are widely classified as "observation" and "perturbation" attacks. Observation or side channel attacks (SCA) consist in observing physical emanations of the system, like power (Differential Power analysis, or DPA [23]) or E/H field (ElectroMagnetic Analysis, or EMA [31]). Thereafter statistical tools are deployed to find dependency between the predicted and observed behavior. Perturbation or fault attacks consist in the injection of faults during the execution of a cryptographic algorithm [7]. From the knowledge of one or multiple couples \{correct ciphertext, faulted ciphertext\}, some hypotheses on the secret key can be discarded. This generic attack strategy is referred to as DFA (Differential Fault Analysis). DFA is very effective against some cryptographic algorithms. For example in AES, the number of faulty ciphertext required to break the key can be as low as two ${ }^{1}$. There are several techniques known for fault injection in a system [21]. The variations of the supply voltage, overclocking, temperature increase, or the irradiation by a laser beam will most probably lead to a wrong computation result that can be exploited to realize a DFA. This kind of attack represents a real threat for the implementation of cryptographic algorithms such as the AES.

In the sequel, and without loss of generality, we focus on the 128-bit version of AES. AES is a substitution permutation network (SPN) product block cipher. It has an iterative structure, consisting in the repetition of ten rounds which are applied to the 16 bytes data block to be encrypted. The 16 bytes are laid out as a matrix of four columns of four bytes $s_{i, j}$, where $0 \leq i \leq 3$ and $0 \leq j \leq 3$. A round consists of a fixed sequence of transformations. Apart from the first and the last rounds, the other eight rounds are identical and consist of four transformations each. The first and last rounds

\footnotetext{
${ }^{1}$ Recently, some even stronger attacks have been presented [40]; the idea is to exploit further relationships from the key scheduling logic to end up with a Boolean equation system that solves the key with one fault, albeit at the cost of more computation. For the need of our HTH design, both types of DFA would be equally adequate.
}

are incomplete, so as to ease the decryption. The four round transformations are called SubBytes, ShiftRows, MixColumns and AddRoundKey.

Piret's DFA [29] assumes only a random "byte-flip" in the last or antepenultimate encryption round. This means that the state state 9 at the 9th round (in the case of AES-128), is replaced by state $\oplus \epsilon$, where $\epsilon$ is a 16 byte vector where only one byte is nonzero:

$$
\text { state }_{9} \stackrel{\text { fault injection }}{\longrightarrow} \text { state }_{9} \oplus \epsilon .
$$

It is an attack that consists in solving a system of nonlinear equations with Boolean variables: the $128+8$ unknown variables are the key and the fault, whereas the $128+128$ known variables are the correct and the faulted ciphertexts. In fact due to the linearity of the MixColumns transformation there are $255 \times 4$ possible differences at the output of the MixColumns. If we suppose a fault on one input byte of the last MixColumns it will affect 4 bytes of the output as shown in Fig. 2 and if we use a pair of correct ciphertext $\mathrm{C}$ and a faulty ciphertext $\mathrm{F}$, we can make a guess about 4 bytes in the key using Piret's algorithm [29]. Eight single faults, located by pairs in the four columns before the MixColumns stage of the ninth round (further referred to as "Round 9") permits the unveiling of the whole key. Alternatively, for this attack, only two single faults located before the MixColumns of the eighth round (further denoted as "Round 8") allows the attacker to find the 16 bytes of the key.

\section{B. DFA-based AES Hardware Trojan Horse}

Fig. 3 shows an example of a HTH introduced in an AES coprocessor. The inserted HTH aids the attacker to perform faults compliant with Piret's DFA. It has three activation conditions:

1) First of all, an external switch (optional) should be activated; it allowed us to validate the design without and with the HTH, simply by moving a sliding lever of a DIP switch on a SASEBO-GII board (for details, please refer to appendix A).

2) Second, the HTH tests that the round counter is equal to 8. As already mentioned, in this round, the fault is scattered to all of the 128 bits of the ciphertext, and these faulted bits allow to retrieve the complete last round key.

3) The third activation condition is a pattern detected in the plaintext. Concretely, our HTH is generic: depending on a parameter $N, N$ bits of the plaintext are tested for equality to one. Such genericity makes it possible for a trade-off: the larger $N$, the smaller the activation probability $\left(1 / 2^{N}\right)$ - by functional test for instance but the larger the HTH (its size grows roughly speaking proportionally with $N$ ). A working example of this HTH on FPGA is shown in Appendix A for $N=16$.

The HTH payload is a single XOR gate which faults one bit of the AES in the inner eighth round. As a consequence, this HTH is more compact than other HTH that would (for instance) output one round key instead of the ciphertext; such strategy requires at least 128 multiplexers. 


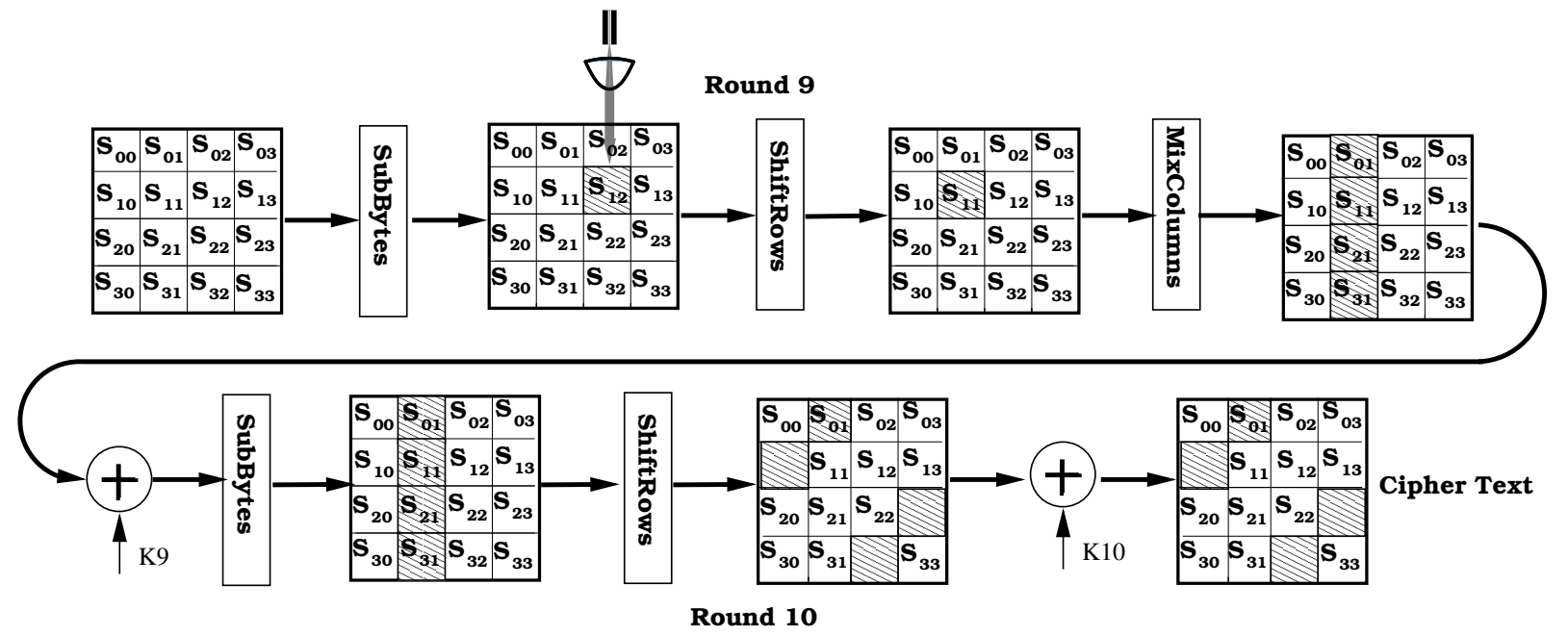

Figure 2. Effect of a fault in last but one round of AES-128.

In our setup, the attacker needs to activate the HTH for 2 encryptions to retrieve the whole key. The power of the attacker will be determined by the HTH activation circuit. The smaller the activation circuit (Trigger) is applied, the more difficult it is to detect HTH, thus the more dangerous it is.

We stress that some interpretation (in other words, little reverse-engineering) of a GDSII file is needed to identify the resources where to plug the HTH. However, we assume that the localization of the state register (128 flip-flops) is fairly easy, and thus understanding roughly speaking the organization of the layout should be feasible without too much pain.

To some extent, the principle of this fault-based HTH is the equivalent to the "intentionally leaky" blocks discussed in [22] for side-channel analyses, but in the domain of fault injection attacks. Indeed, the side-channel (resp. fault injections) attacks are used for a constructive feature (although our HTH actually implements a malicious functionality!).

Finally, let us mention that, to our best knowledge, the first HTH that relies on the insertion of a distant fault injection mechanism is the "bug attack" [6]. It consists in slightly altering a hardware integer multiplier, in such a way it delivers always the correct answer but for at least one configuration of the inputs. It has been shown by Biham, Carmeli and Shamir that this permits a remote Bellcore [9] attack that allows, for the insider who inserted the HTH and under some circumstances (e.g. depending on the padding scheme), a retrieval of the private key. The HTH presented in this section is based on a similar idea that allows a remote DFA on symmetric block ciphers.

\section{Text-Only DFA-Based Hardware Trojan Horse}

The Hardware Trojan Horse described in the previous section II-B might seem awkward. Indeed, its trigger condition requires the conjunction of several events, including a specially crafted plaintext and an explicit binary activation. This choice had been motivated by the ease to test it on the SASEBO board. Nonetheless, it is possible to devise a hardware Trojan horse which does not require the binary activation input, while still allowing for a key extraction by DFA. One first method simply consists in designing the Trojan so that it is sequential. For instance, its trigger condition can be as follows:

- If the plaintext has such distinctive characteristic, then

- inject a fault in the antepenultimate (i.e., last but two) round of the next encryption.

The attacker then simply sends twice the same plaintext with the activation condition. The first one will be encrypted correctly, while the second one will be corrupted. Hence a DFA attack is possible.

An alternative solution is to design an AES block which is able both to encrypt and decrypt. The Trojan activates after the second round (i.e., mirror to the antepenultimate round) of decryption, when some specially crafted ciphertext is submitted. Let us denote one of such ciphertexts as $c$. The (faulted) decryption yields an erroneous plaintext $p^{\prime}$. However, owing to the invertibility of the AES rounds and of the fault injection (modeled as an exclusive-or with the state recall Eqn. (1)), it can also be considered that $c$ is the faulty ciphertext associated with the encryption of the plaintext $p^{\prime}$, which unfolded without error until round nine (as for AES128), where a fault is injected. Thus, it suffices for the attacker to request the encryption of $p^{\prime}$, which will execute without fault (since the Trojan is only active in decryption mode). This encryption yields $c^{\prime}$, the correct ciphertext associated with plaintext $p^{\prime}$. Therefore, the pair $\left(c, c^{\prime}\right)$ allows to mount a DFA attack.

Still, we notice a limitation. Both solutions have a drawback: they do not work in the presence of some modes of operation, because the inputs of the (trapped) AES cannot be chosen. 


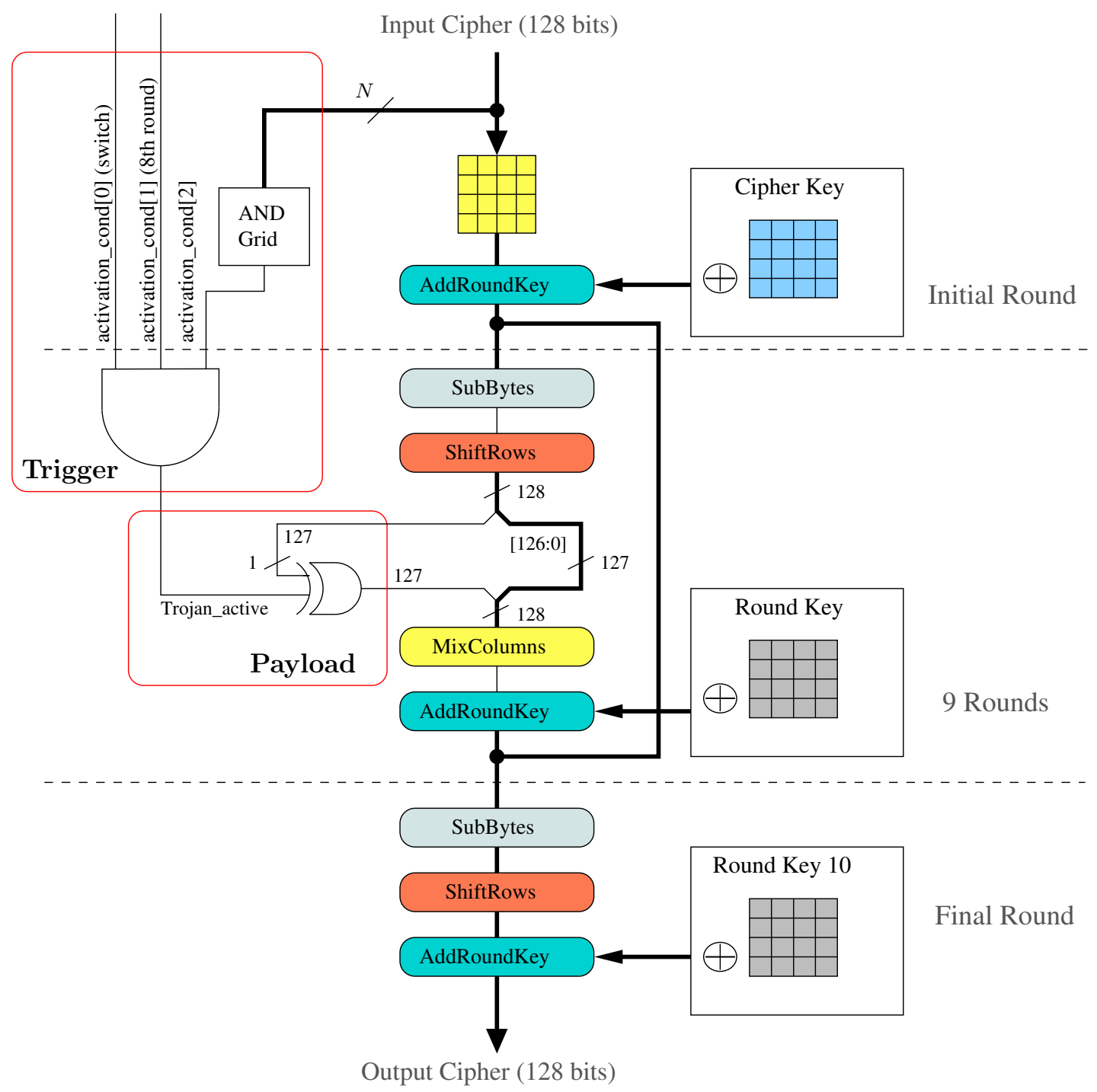

Figure 3. Differential Fault Analysis (DFA) based HTH in AES - Illustration of a remote DFA enabled by the HTH

III. Low-Cost Hardware Trojan Horse Detection by GDSII / Picture COMParison

\section{A. Reverse Engineering}

Reverse engineering is often used in the semiconductor industry to extract technical or patent related information from a competitor's chip [18]. The chip can be reverse engineered at different levels like: product components, systemlevel, process-level and circuit-level [39]. In our case we are interested with the circuit-level reverse engineering. Modern devices are fabricated in technology like $45 \mathrm{~nm}$ or lower which can have up to 12 layers of metal and several millions of transistors. The detail of the circuit are extracted in the following sequential steps:

- package removal;

- delayering;

- imaging.
In the state of the art, "destructive reverse-engineering" can be used to detect HTH for our scenario. Reverse-engineering is generally performed by Chemical Metal Polishing (CMP) followed by Scanning Electron Microscope (SEM) image reconstruction and analysis [38]. It helps to reconstruct exactly all via, metal and silicon layers. After, the determination of the "correctness" of a chip is performed through visual comparison with a known good example or "golden reference". But this technique is very expensive since it takes a lot of time (hence costs a lot) to realize properly: the mere error in a picture (because the delayering left pieces of material on the chip surface or because the recognition software [34] failed) makes the reverse engineering fail. In addition, modern devices are extremely small and densely packed, which makes the cost (in money and time) of reverse engineering even higher. 


\section{B. Medium Cost Hardware Trojan Horse Detection}

In this paper, we demonstrate that HTH inserted at GDSII level can be spotted with a visual detection technique. The main argument is that the full reverse-engineering is overkill to detect a change in a layout. Mesoscopic imaging (obtained with a $\times 150$ optical microscope) is enough to conduct a coarse imaging comparison between the GDSII file submitted for fabrication and the returned circuit. High-end visible-light microscope and camera are termed specialized equipments in the attacks quotation application note [15, §3.7] (for Common Criteria [14]), which means that they considered medium cost laboratory tools. Indeed, the low-level (masks drawing) "bitmap" comparison of two images is a more elementary task than a full-fledged reverse engineering (that in addition aims at interpreting the layout, i.e. reconstruct the netlist, by giving sense to the picture). Still said differently, reverse engineering is well suited to perform an "image versus netlist" comparison, whereas we research in this paper to make a sheer "image versus layout" comparison.

By optical observation, the M6 shape (topmost layer) can be recovered. For example, Fig. 4 shows the layout and the picture of the fabricated chip side-by-side. The layout is opened in Mentor Graphics Calibre Litho View tool (r21024.25 ), because it allows to set the color and the transparency level for each layer. Specifically, we decided to represent the topmost metal layer with a solid color, as this corresponds to the non-transparency of this layer by optical microscopy. The pictures were taken by a confocal microscope with a $\times 150$ lens, and are presented in real colors (i.e. no image edition has been performed: the image is raw). In the picture view, the $L$ shapes (that appear "white") are aluminium dummy fillings ${ }^{2}$. added to meet the density constraints of design rules. These (innocuous) shapes cannot be seen in the layout view because they are added by the silicon founder at masks preparation steps. The technological node is $130 \mathrm{~nm}$, where the metal lines are in copper (that appear in "orange" color). The M6 lines are rather routed vertical (but for the ring). Their smallest width is $460 \mathrm{~nm}$. To a lesser extent, the M5 lines can be seen: those are thiner lines (100 nm large). The $L$ shapes slightly mask M6 / M5 layers, but still the lines can be seen. In the "side of the module" area of Fig. 4, various power and ground rings are visible. In the "middle of the module" area of Fig. 4, power strips and some signals of the AES core are visible. Some of them are very noticeable, as they are making turns or even zigzags (i.e. multiple jogs). If the chip has a shield layer (refer to [10]), then the image recognition might be harder.

Our experiments are low cost, insofar as the circuits are not depassivated (hence images are slightly blurred). Notwithstanding we see that the pictures and the GDSII remarkably match. Therefore, any single modification of the M6 (or M5) routing could be easily detected visually.

In addition, the analyzed circuit remains functional, and so it can be trusted alone. Indeed, in the case where both circuits with and without HTH are produced, then the absence of HTH

\footnotetext{
${ }^{2}$ Given the orientation of the picture, each $L$ shape appears like a $\Gamma$ symbol.
}

Table I

USAGE OF ROUTING RESOURCES PER LAYER FOR THE AES CASE-STUDY WITH 95\% CORE UTILIZATION RATE

\begin{tabular}{|c||c|c|c|c|c|c|}
\hline Density & $M 1$ & $M 2$ & $M 3$ & $M 4$ & $M 5$ & $M 6$ \\
\hline$N=0$ & $44 \%$ & $22 \%$ & $20 \%$ & $17 \%$ & $10 \%$ & $5 \%$ \\
$N=1$ & $44 \%$ & $22 \%$ & $20 \%$ & $17 \%$ & $10 \%$ & $5 \%$ \\
$N=2$ & $44 \%$ & $22 \%$ & $20 \%$ & $17 \%$ & $10 \%$ & $5 \%$ \\
$N=4$ & $44 \%$ & $22 \%$ & $20 \%$ & $17 \%$ & $10 \%$ & $5 \%$ \\
$N=8$ & $44 \%$ & $22 \%$ & $20 \%$ & $17 \%$ & $10 \%$ & $5 \%$ \\
$N=16$ & $44 \%$ & $22 \%$ & $20 \%$ & $17 \%$ & $10 \%$ & $5 \%$ \\
$N=32$ & $44 \%$ & $22 \%$ & $20 \%$ & $17 \%$ & $10 \%$ & $5 \%$ \\
$N=64$ & $44 \%$ & $22 \%$ & $20 \%$ & $17 \%$ & $10 \%$ & $6 \%$ \\
$N=128$ & $44 \%$ & $22 \%$ & $20 \%$ & $18 \%$ & $11 \%$ & $6 \%$ \\
\hline
\end{tabular}

on one circuit cannot prove the absence of HTH on all the circuits.

Now, if we extrapolate to technologies where the feature size is smaller (i.e. $<130 \mathrm{~nm}$ ), a visual inspection might be more delicate, and probably more error-prone. This is why in the next section IV we use a statistical tool (viz. the crosscorrelation) instead of the exact shape recognition.

It can be argued that it is possible for the designer of a HTH to route it using the inner metal layers $(<\mathrm{M} 6)$. For small HTHs, this is certainly true. However, when the HTH becomes larger and larger, then low-level metal layers (M1, $\mathrm{M} 2$, etc.) are already very crowded, and thus will feature a faster congestion. For instance, in our AES module, the routing density per layer for a core utilization rate of $95 \%$ is given in Tab. I. For reasonable size hardware HTHs, the higher metal layers are more likely to be used for routing the HTHs. Tab. I also shows for AES infected with HTHs of size $N=1$ to $N=128$. Obviously, some parts of the lower metal layers are also used for routing. For the success of our method of visual detection, it is sufficient that the higher layers are used for routing some part of the HTHs. The higher the layer used for routing the HTHs the simpler is it detection. Therefore if only a small part of the HTHs uses the M6 tracks for routing, our method can detect the presence. We do not need to go down the other layers. But if the HTH is routed in lower level metals, then circuit must be delayered. This operation is more costly, however, since the passivation has been removed, the layout stands out more clearly.

As a consequence, the strategy of an attacker is to make the HTH as furtive as possible. Typically, she cannot change the dimension of the IP block, because this will be the first check made by the designer. So, the attacker must meet the challenge to interleave the HTH gates and interconnections signals inside the existing layout. Moreover, to be as discreet as possible, low-level metal layers should be preferred.

However, as will be argued in the next section (and quantified with similarity metrics), it is extremely difficult to insert logic (even some a couple of tens) in a design that is already compact (density over $80 \%$ ) without changing the appearance of the layout. 

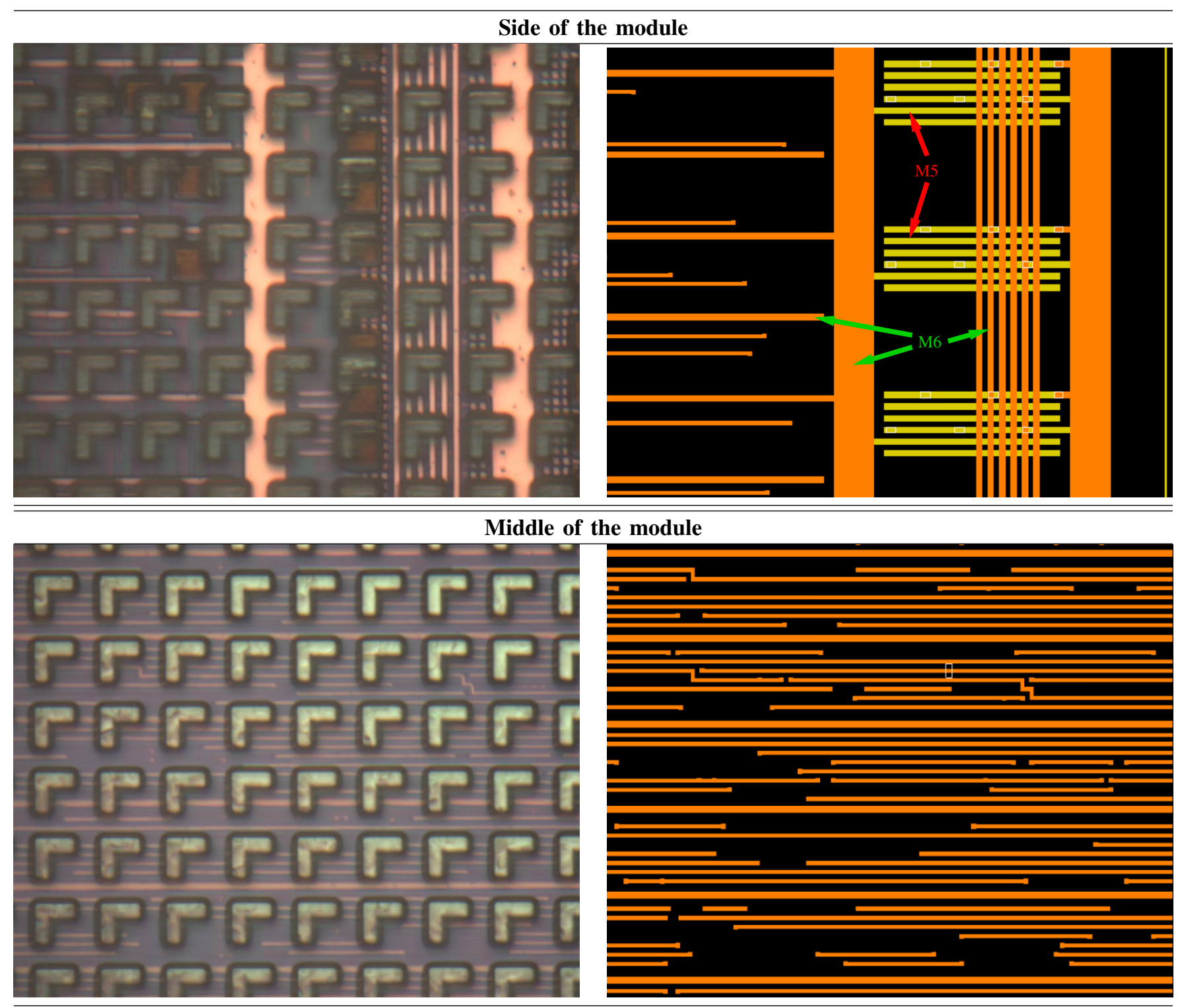

Figure 4. Picture (left hand side) and GDSII layout (right hand side - M6 in orange for Middle and Side of the module, M5 in yellow for Side of the module) of an AES-128 cryptographic accelerator (area size: $67 \mu \mathrm{m} \times 60 \mu \mathrm{m}$ )

\section{AES Hardware Trojan Horse Layout Study}

\section{A. Experiment Setup}

In this experiment, we evaluate the feasibility and impacts of HTH insertion at layout level. First, we create a circuit layout without HTHs and then we insert a HTH with different sizes in this layout while maintaining the IC placement and the routing. After, we calculate the cross-correlation between these layouts (layout without HTHs and layout with HTHs inserted) to see the impacts of HTH insertion. The Cross-Correlation $C C$ is defined as the measure of similarity between two waveforms or processes $f_{1}$ and $f_{2}$. It can be expressed as:

$$
C C=\Sigma_{x, y} f_{1}(x, y) \cdot f_{2}(x, y)
$$

However, for image processing, cross-correlation may not be an optimal metric. The problem arises from factors like light- ning and exposure conditions. In such cases, the Normalized Cross-Correlation $(N C C)$ is recommended, which normalizes the images before applying correlation. It is expressed as:

$$
N C C=\frac{1}{n_{x} \cdot n_{y}} \cdot \frac{\Sigma_{x, y}\left(f_{1}(x, y)-\overline{f_{1}}\right) \cdot\left(f_{2}(x, y)-\overline{f_{2}}\right)}{\sigma_{f_{1}} \cdot \sigma_{f_{2}}},
$$

where $n_{x}$ (resp. $n_{y}$ ) is the number of $x$ (resp. $y$ ) values. NCC is relevant to compare two heterogeneous objects (e.g. image versus GDSII), unlike the "difference" that applies only to two homogeneous objects (e.g. GDSII versus GDSII). We also introduce another notion i.e. Reverse NCC or RNCC expressed as:

$$
R N C C=1-|N C C| \in[0,1] .
$$

The sole purpose of defining $R N C C$ is for better visibility of results. If the $N C C$ between the image and the layout is low 


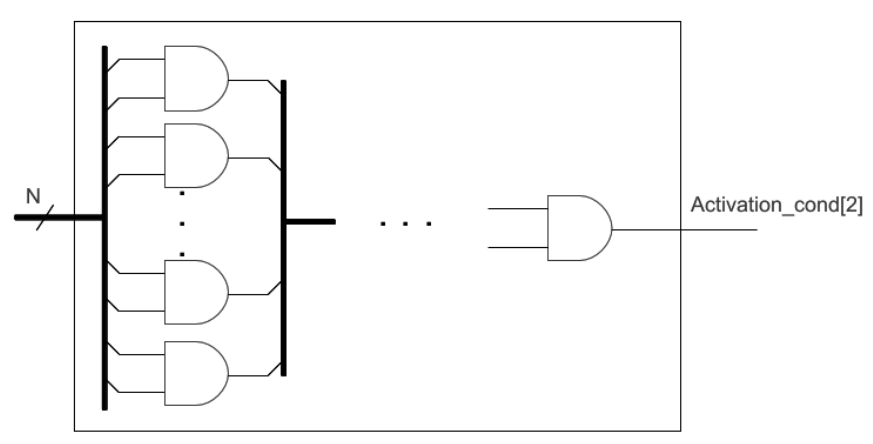

Figure 5. HTH with $N-1$ AND gates (AND tree) inserted in AES circuit

then $R N C C$ will be high and it will be easy to distinguish the error.

An AES 128-bit algorithm, as shown in Fig. 3, is implemented to perform this experiment. Different AES layouts with different core utilization rates are generated thanks to Cadence Encounter (product SOC version 2012/8.1) software. Core utilization rate is the area occupied by the standard cells of the AES circuit relative to the total area of silicon. For each core utilization, 8 HTHs with different sizes are inserted. The structure of the HTH "trigger" consists in a series of AND gates, as depicted in Fig. 5. The size of HTH is the number of AND gates which varies from 1 gate to 128 gates. These HTHs will observe the $N$ inputs of the AES circuit and inject a fault to the 8th AES round when the corresponding inputs are in the high state. Of course we are aware that less than $(N-1)$ AND gates might be needed to observe $N$ inputs (because of optimization with complex gates and use of inverted NAND gates instead of AND gates), but we use a 2 input AND tree to have a regular structure. It has the advantage of growing linearly with the number of input bits observed, which allows for a simple law to quantify the impact of HTH size on the AES layout. The next section shows the result of this experiment.

To maintain the placement and the routing of AES circuit, we use the "ECO Placement" function of Encounter software. The "ECO" option (short for "Engineering Change Order") is a local modification, customarily used to fine-tune a design before tape-out. It allows to modify a couple of gates / nets, without affecting the rest of the circuit, considered acceptable. First, this function verifies the layout (with Design Rule Checking, aka DRC). Second it inserts standard cells of HTHs in unused places in the circuit. At the end, it routes all HTH nets while minimizing the modification created to original layout. The core utilization rates used are 50\%, 60\%, $70 \%, 80 \%, 90 \%, 95 \%$ and $99 \%$. Eight different HTHs used are 1, 2, 4, 8, 16, 32, 64, 128 AND gates HTHs. In total, 63 different AES layouts were generated thanks to this software (7 original layouts for each core utilization rate and 56, 8 for each core utilization rate (CUR), modified layouts).

\section{B. Experiment Results}

In reality, we can see only the last metal and isolation layers in the pictures taken by microscopy. Because of this, for the first experiment, all AES layouts generated show only the 6th metal layer (the last metal layer in our case) and hide others layer. Fig. 6(a), 6(b) and 6(c) show the original AES, and two modified AES layouts (with 1 AND gate and 128 AND gates HTHs) for 50\% CUR. Visually, there is very little difference between these layouts. By using cross-correlation calculations, we can extract their differences.

The Tab. II indicates all NCC between these layouts. Each line shows $N C C$ between AES with HTH and original layouts for the corresponding core utilization rate. Note that these coefficients are the comparisons between GDSII files so they are calculated in the best cases. This result shows that $N C C$ decreases when core utilization rate and HTH size increases. We realize that for CUR more than $80 \%$ and for HTHs more than 64 gates, NCC decreases by about $10 \%$ or more. So these HTHs can be detected. In this experiment, 64 gates are equivalent to about $0.5 \%$ of AES circuit which is composed of 12, 267 standard cells. The values in bold correspond to the cases when "ECO placement" function cannot place and route HTHs without making errors in the layout. In these cases, we must replace and reroute of all standard cells (AES and HTH standard cells). This causes a big difference compared to the original layout ( $N C C$ equal to $\mathbf{0 . 4 0 1 0} \& \mathbf{0 . 3 7 9 8}$ ) and so the resulting HTH insertion is easy to detect. Values " $\mathbf{X}$ " mean that even with replace and reroute of all standard cells, Encounter cannot achieve the layout without errors.

The fact that "ECO placement" is able to place an HTH of 128 gates when CUR is $95 \%$ but fails for $90 \%$, is a property specific to our design and not at all generic. Probably this would not hold for another design; actually, we expect that the $N C C$ decreases with the CUR (provided we could estimate the $N C C$ over a large number of circuits).

In addition, at the cost of depassivation and of the dissolution of the oxide layers ${ }^{3}$, it becomes possible to observe from the top the superimposition of all the metal layers [30]. With such a preparation, the HTH will obviously be spotted more accurately. We recomputed the cross-correlation under this assumption. This time, all AES layouts (GDSII files) show all metal layers. Fig. 7(a), 7(b) and 7(c) show the original AES, and two modified AES layouts (with 1 AND gate and 128 AND gates HTHs) for 50\% CUR. The Tab. III indicates all $N C C$ coefficients between these layouts. By observing Tab. II and Tab. III, we notice that NCC coefficients for 6th metal layer AES layouts are better. This is expected because adding all metal layer into $N C C$ computation is equivalent to adding noise to the computation. For a strong attacker who can restrict the HTH to lower metal levels, the computations for all metal layers layout can be applied for detection.

We also compute the pixel-wise difference between the two

\footnotetext{
${ }^{3}$ Those oxide layers are also known as "inter-level dielectric" (ILD) or simply "passivation layers".
} 


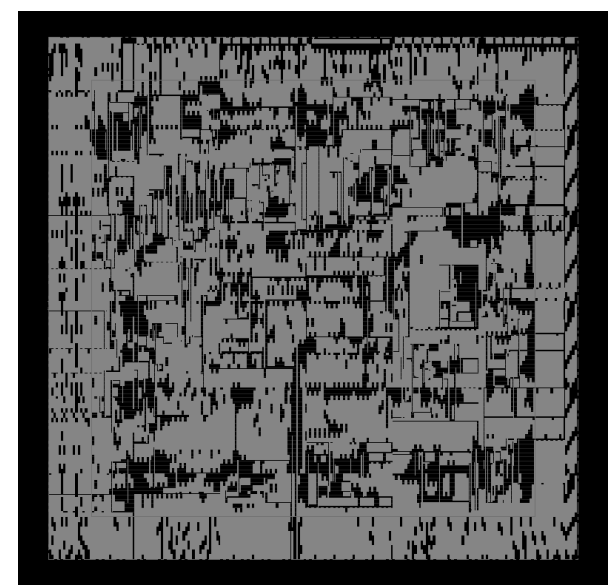

(a)

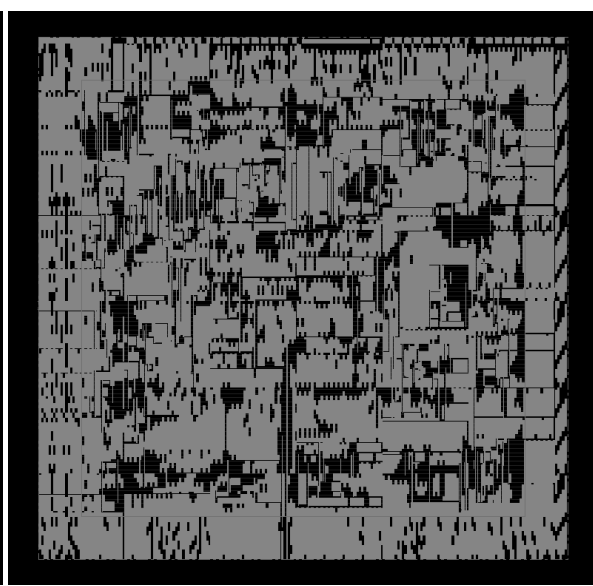

(b)

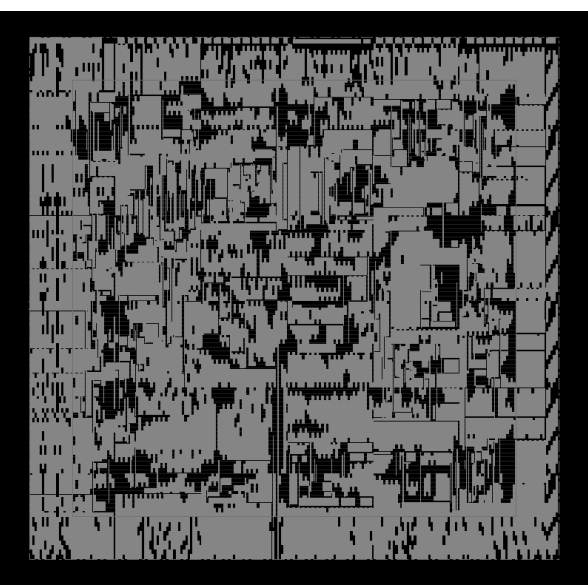

(c)

Figure 6. 6th metal layer AES layouts $(1200 \mu \mathrm{m} \times 1200 \mu \mathrm{m})$ with $50 \%$ core utilization rate for (a) Original AES, (b) AES with 1 AND gate HTH, (c) AES with 128 AND gate HTH

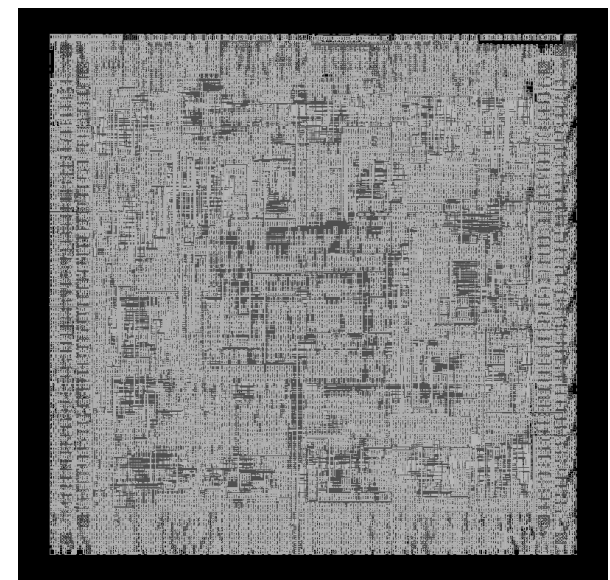

(a)

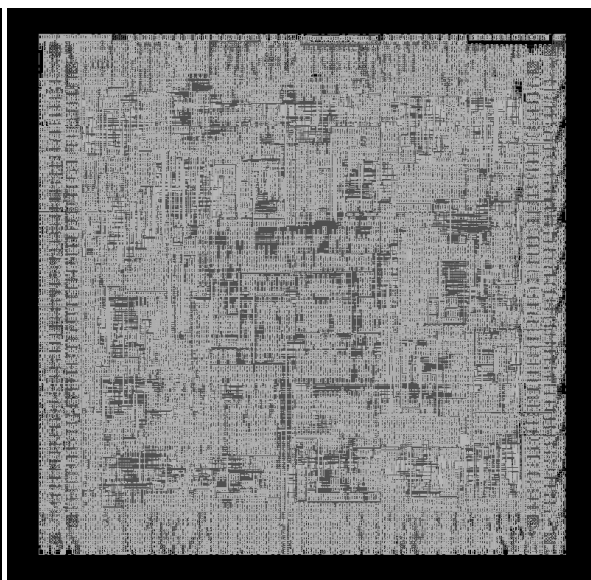

(b)

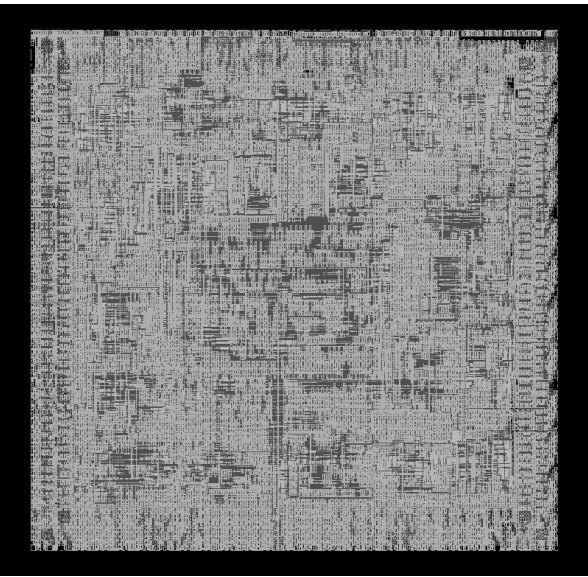

(c)

Figure 7. AES layouts $(1200 \mu \mathrm{m} \times 1200 \mu \mathrm{m})$ with $50 \%$ core utilization rate for (a) Original AES, (b) AES with 1 AND gate HTH, (c) AES with 128 AND gate $\mathrm{HTH}$

images. The Fig. 8(a) and 8(b) show the differences of all metal layers AES layout with 1 and 128 AND gates HTHs compared with original AES layout for $50 \%$ core utilization rate. So Fig. 8(a) is the difference between Fig. 6(a) and Fig. 6(b), and Fig. 8(b) is the difference between Fig. 6(a) and Fig. 6(c). We notice that these differences are small but they are concentrated in areas where the HTH standard cells are actually placed. Following this observation, we realize a grid-wise RNCC calculations for these layouts. The principle is to split into several pictures of same size using a grid. Each sub-picture of a given layout is then correlated with the corresponding sub-picture of the reference layout. Grid-wise RNCC permits not only to detect the trojan but also localise it visually. The Fig. 9(a), 9(b), 10(a) and 10(b) show the grid-wise $R N C C$ of the AES HTH layout (1 and 128 AND gates) for $50 \%$ and $95 \%$ core utilization rates. For these calculations, layouts are cut in 32 parts horizontally and in 31 parts vertically. So there are in total $32 \times 31=992$ pieces and also 992 correlation coefficients without overlap for each comparison. Note that these coefficients are reverse correlation coefficients (i.e. 1 - cross-correlation coefficients) for being seen better in the figures. We notice that the cross-correlation coefficients were improved. HTH standard cells can be also localized with this technique and also be detected easier (see in [24] how to prepare a circuit accordingly with HF).

\section{Validation on a Real Trojan Horse Insertion}

In order to validate the relevance of $N C C$ as a tool to measure the similarity between an observed image and a reference layout, we actually tested this metric in the real context of HTH recognition. Figure 11 shows two GDSII layouts, one infected and the other genuine.

Obtaining an infected GDSII is not a practical assumption. Fabricating a golden reference circuit in an trusted fab is neither a practical assumption. Thus, the detection shall be 
Table II

NCC Coefficients between 6th Metal layer of Original and Infected AES layouts

\begin{tabular}{|l|c||c|c|c|c|c|c|c|c|c|}
\hline \multicolumn{2}{|c||}{} & \multicolumn{9}{c|}{ HTH size (Nb of AND gates) } \\
\cline { 2 - 9 } \multicolumn{1}{|c|}{} & 1 & 2 & 4 & 8 & 16 & 32 & 64 & 128 \\
\hline & $50 \%$ & 0.9991 & 0.9972 & 0.9981 & 0.9950 & 0.9933 & 0.9918 & 0.9815 & 0.9668 \\
& $60 \%$ & 0.9987 & 0.9968 & 0.9959 & 0.9955 & 0.9944 & 0.9893 & 0.9788 & 0.9670 \\
& $70 \%$ & 0.9989 & 0.9981 & 0.9918 & 0.9941 & 0.9881 & 0.9850 & 0.9594 & 0.9067 \\
& $80 \%$ & 0.9999 & 0.9965 & 0.9898 & 0.9957 & 0.9780 & 0.9711 & 0.8970 & 0.8509 \\
& $90 \%$ & 0.9988 & 0.9990 & 0.9983 & 0.9962 & 0.9832 & 0.9572 & 0.8858 & $\mathbf{0 . 4 0 1 0}$ \\
& $95 \%$ & 0.9997 & 0.9984 & 0.9980 & 0.9889 & 0.9589 & 0.9115 & 0.8824 & 0.8202 \\
& $99 \%$ & 0.9917 & 0.938 & 0.9714 & 0.9527 & $\mathbf{0 . 3 7 9 8}$ & $\mathbf{X}$ & $\mathbf{X}$ & $\mathbf{X}$ \\
\hline
\end{tabular}

Table III

NCC Coefficients between all metal layers of Original and Infected AES layouts

\begin{tabular}{|l|c||c|c|c|c|c|c|c|c|c|}
\hline \multicolumn{2}{|c||}{} & \multicolumn{9}{c|}{ HTH size (Nb of AND gates) } \\
\cline { 3 - 10 } & 1 & 2 & 4 & 8 & 16 & 32 & 64 & 128 \\
\hline & $50 \%$ & 0.9996 & 0.9788 & 0.9794 & 0.9770 & 0.9766 & 0.9760 & 0.9719 & 0.9708 \\
& $60 \%$ & 0.9993 & 0.9975 & 0.9973 & 0.9970 & 0.9964 & 0.9923 & 0.9843 & 0.9783 \\
& $70 \%$ & 0.9991 & 0.9987 & 0.9947 & 0.9952 & 0.9924 & 0.9889 & 0.9655 & 0.9272 \\
CUR (Core Utilization Rate) & $80 \%$ & 0.9997 & 0.9976 & 0.9942 & 0.9969 & 0.9868 & 0.9790 & 0.9291 & 0.8915 \\
& $90 \%$ & 0.9990 & 0.9988 & 0.9981 & 0.9964 & 0.9878 & 0.9709 & 0.9255 & $\mathbf{0 . 5 1 6 2}$ \\
& $95 \%$ & 0.9995 & 0.9978 & 0.9974 & 0.9927 & 0.9742 & 0.9387 & 0.9159 & 0.8661 \\
& $99 \%$ & 0.994 & 0.9965 & 0.9803 & 0.962 & $\mathbf{0 . 4 9 0 5}$ & $\mathbf{X}$ & $\mathbf{X}$ & $\mathbf{X}$ \\
\hline
\end{tabular}

based only of the comparison between the genuine GDSII and the fabricated chip, that may (or not) contain a HTH.

If the untrusted chip contains a HTH, the $N C C$ value will indicate its presence to the designer. To validate our concept, we perform the following operations.

1) We fabricated a chip which contains a very tiny $\mathrm{HTH}$ (whose impact at the M6 topmost metal level is only to add two routing wires) and thus we have its GDSII.

2) Since we also know the HTH, we can obtain the genuine GDSII from the trojaned GDSII.

3) Then we take a photo of the chip placed under an optical microscope achieving a magnification zoom of $150 \times$.

4) Finally, we compute the NCC between the photo of the real trojaned chip and the two GDSII available (Fig. 11).

The NCC with a picture (the same as in middle of the module part of Fig. 4, but rotated for the ease of presentation) is computed:

- it is $1.56 \%$ between the trojan infected GDSII (unknown to the designer, since forged at the silicon foundry) and the image,

- and only $0.67 \%$ between the original GDSII (known by the designer) and the image.

We emphasize again that obtaining a trojan infected GDSII is not very realistic. Therefore a designer cannot know the largest cross-correlation that can be obtained between the trojaned GDSII (corresponding to the picture) and the picture. However, the grid-wise NCC method can detect the hardware trojan horse in such cases. It is expected that the grids that are not infected by the trojan, will yield about
$1.56 \% N C C$ on the layout versus image comparison. Only the grids with a significant lower $N C C(0.67 \%)$ will raise a concern. Thus, the local variation of cross-correlation, of order $(1.56-0.67) / 1.56=57 \%$, will allow to signal the presence of a HTH in the design; indeed, $57 \%$ is a large contrast. It is important to have such a large contrast, because eventually, the grid-based correlation will be noisy, for several reasons:

- the photographic picture can be blurry,

- the correlation values in areas without HTH might depend on the contents of this very area,

- obstacles, like the superimposed $L$ shapes in M7, might not be evenly distributed over the grid.

However, if the M7 L shapes are clearly identifiable, the contrast can be increased by removing (i.e. by replacing them by black areas) from both the picture and the layout.

To further investigate, the designer can then focus on the infected area by repeating the same detection technique with magnified pictures (of local SEM imaging) to attest the presence of the HTH.

\section{RECOMMENDATIONS}

During this experiment, we realize that HTHs insertion at layout level is not easy for the attacker. Firstly, he must understand the functionality of the circuit from the GDSII file. Secondly, he must keep the original layout mainly unchanged, otherwise a "forensic" visual inspection is very likely to detect the HTH. Thus the HTH must be placed-and-routed inbetween the (unaltered) existing circuit. Results, given in Section IV, show that HTHs insertion become more and more difficult when core utilization rate increases. We recommend 
the designer to use more than $80 \%$ core utilization rate (CUR) for his design. From this CUR, the insertion of HTH is very unlikely because of HTH size. If the HTH size is small, this HTH can be accidentally activated and hence can be easily detected at test time. If the HTH has a reasonable size (for example superior to 64 gates in our study case), it is visually detected (NCC decreases by about $10 \%$ or less for a CUR superior to $80 \%$ ). A similar observation was proposed in [42], where the authors recommended to avoid dead space in an IC in order to prevent HTH insertion. We reconfirm the same observation and take it a bit further in order to quantify it in terms of core utilization rate. With this, either the HTH insertion fails (because it is too large w.r.t. the available placeand-route resources ${ }^{4}$ ), or its insertion can be detected with a medium cost visual technique. Further, our criteria based on the CUR is "global" to a design, and can easily be set as a constraint in all $\mathrm{P} \& \mathrm{R}$ tools.

\section{Conclusion and Perspectives}

In this paper, we have presented the feasibility and impacts of HTHs insertion to the circuit layout (especially for AES circuit). Additionally, we have demonstrated the possibility to detect HTHs with a medium cost visual technique; this technique can be automated thanks to a cross-correlation. In particular, the observation of the sole top-level metal layer suffices (for large enough HTHs); thus the method avoids mechanical or chemical preparation, known to produce dust of material that would cause many false positive detections. As a corollary, if no HTH is detected in the observed circuit, then this circuit can be trusted and used safely in an application, even if other circuits from a different batch happen to be infected by a HTH. The results show that the insertion complexity and the visibility of HTH increases with the core utilization rate of circuits. With a high CUR, HTH can be detected by comparing layout images and GDSII file. This technique cannot detect HTH inserted in specification or RTL level but it can be combined with others detection techniques to ensure the security of the full fabrication process.

Checking for the genuineness of an observed picture (such as the lefthand side of Fig. 4) against a known template (such as the layout at the righthand side of Fig. 4) can benefit from better decision strategies than a simple correlation. Indeed, the layout is rich of particular features. Thus, for instance, minutæ analyses for fingerprint recognition can be used advantageously (using routing zigzags for instance); those techniques are also interesting insofar as they allow to tune the false acceptance/rejection rate(s). However, application of minutæ-like features in context of HTH is an open research topic. In our case, as we do not want to accept as genuine a trojaned circuit, we intend to configure the technique to minimize its false acceptance rate.

\footnotetext{
${ }^{4}$ In particular, our experiments with the Cadence Encounter place-and-route tool show that even if there is "physically" some room for the insertion of the $\mathrm{HTH}$, the routing-aware placer and the router itself can fail. This is because space on the die is not the limitating factor for the $\mathrm{HTH}$, but rather the availability of free routing tracks.
}

Besides, we could also profit of advanced techniques such as those used in steganalysis to detect a piece of information (the HTH) hidden in noise (the genuine layout).

\section{ACKNOWLEDGMENTS}

This project has been funded by the French Government, under grant FUI \#14 HOMERE (Hardware trOjans : Menaces et robustEsse des ciRcuits intEgrés). We acknowledge interesting inputs from the other members of the consortium, in particular Julien Francq (Cassidan Cyber Security) and Denis Réal (DGA). Also, while preparing the camera-ready version of the paper, we appreciated the positive inputs from Nicolas Bruneau, Guillaume Duc, Zakaria Najm, Pablo Rauzy and Youssef Souissi (crypto group of TELECOM-ParisTech). Besides, we are grateful to Yves Mathieu for the setup of the Calibre LITHO view tool, to Antoine Khy and Fabien Thomas-Brans for pictures, and to the CMP [13] staff (especially Kholdoun Torki and Jean-François Paillotin) for an excellent support in the fabrication with STMicroelectronics HCMOS9GP technology and the after-sale maintenance of the SecMat-v2 ASIC [17, Fig. 1]. Eventually, we are grateful to Prof. David Naccache for the suggestion about a DFA-based hardware trojan that would trigger only on some plaintexts.

\section{REFERENCES}

[1] Miron Abramovici and Paul Bradley. Integrated circuit security: new threats and solutions. In Frederick T. Sheldon, Greg Peterson, Axel W. Krings, Robert K. Abercrombie, and Ali Mili, editors, CSIIRW, page 55. ACM, 2009.

[2] Sally Adee. The Hunt For The Kill Switch. IEEE Spectr., 45(5):34-39, May 2008.

[3] Dakshi Agrawal, Selcuk Baktir, Deniz Karakoyunlu, Pankaj Rohatgi, and Berk Sunar. Trojan Detection using IC Fingerprinting. In Proceedings of the 2007 IEEE Symposium on Security and Privacy, SP '07, pages 296-310, Washington, DC, USA, 2007. IEEE Computer Society.

[4] Subidh S. Ali, Rajat Subhra Chakraborty, Debdeep Mukhopadhyay, and Swarup Bhunia. Multi-level attacks: An emerging security concern for cryptographic hardware. In Design, Automation Test in Europe Conference Exhibition (DATE), 2011, pages 1-4, march 2011.

[5] Mainak Banga and Michael S. Hsiao. A Novel Sustained Vector Technique for the Detection of Hardware Trojans. In Proceedings of the 2009 22nd International Conference on VLSI Design, VLSID '09, pages 327-332, Washington, DC, USA, 2009. IEEE Computer Society.

[6] Eli Biham, Yaniv Carmeli, and Adi Shamir. Bug attacks. In CRYPTO, volume 5157 of $L N C S$, pages 221-240. Springer, 2008. Santa Barbara, CA, USA.

[7] Johannes Blömer and Jean-Pierre Seifert. Fault Based Cryptanalysis of the Advanced Encryption Standard (AES) Financial Cryptography. In Rebecca Wright, editor, Financial Cryptography, volume 2742 of Lecture Notes in Computer Science, pages 162-181. Springer Berlin / Heidelberg, 2003.

[8] Gedare Bloom, Bhagirath Narahari, and Rahul Simha. OS Support for Detecting Trojan Circuit Attacks. In Mohammad Tehranipoor and Jim Plusquellic, editors, HOST, pages 100-103. IEEE Computer Society, 2009.

[9] Dan Boneh, Richard A. DeMillo, and Richard J. Lipton. On the Importance of Checking Cryptographic Protocols for Faults. In Proceedings of Eurocrypt'97, volume 1233 of LNCS, pages 37-51. Springer, May 11-15 1997. Konstanz, Germany. DOI: 10.1007/3-540-69053-0 4.

[10] Sébastien Briais, Jean-Michel Cioranesco, Jean-Luc Danger, Sylvain Guilley, David Naccache, and Thibault Porteboeuf. Random active shield. In Guido Bertoni and Benedikt Gierlichs, editors, FDTC, pages 103-113. IEEE, 2012.

[11] Rajat Subhra Chakraborty, Somnath Paul, and Swarup Bhunia. Ondemand transparency for improving hardware trojan detectability. In Mohammad Tehranipoor and Jim Plusquellic, editors, HOST, pages 4850. IEEE Computer Society, 2008. 
[12] Rajat Subhra Chakraborty, Francis G. Wolff, Somnath Paul, Christos A. Papachristou, and Swarup Bhunia. MERO: A Statistical Approach for Hardware Trojan Detection. In Christophe Clavier and Kris Gaj, editors, CHES, volume 5747 of Lecture Notes in Computer Science, pages 396410. Springer, 2009.

[13] Circuits Multi-Projets (CMP). Website: http://cmp.imag.fr.

[14] Common Criteria Consortium. Common Criteria ( $a k a$ CC) for Information Technology Security Evaluation (ISO/IEC 15408), 2013.

Website: http://www.commoncriteriaportal.org/.

[15] Common Criteria. Application of Attack Potential to Smartcards, Mandatory Technical Document, Version 2.7, Revision 1, CCDB2009-03-001, March 2009. http://www.commoncriteriaportal.org/files/ supdocs/CCDB-2009-03-001.pdf.

[16] Jean-François Gallais, Johann Großschädl, Neil Hanley, Markus Kasper, Marcel Medwed, Francesco Regazzoni, Jörn-Marc Schmidt, Stefan Tillich, and Marcin Wójcik. Hardware trojans for inducing or amplifying side-channel leakage of cryptographic software. In Liqun Chen and Moti Yung, editors, INTRUST, volume 6802 of Lecture Notes in Computer Science, pages 253-270. Springer, 2010.

[17] Sylvain Guilley, Laurent Sauvage, Jean-Luc Danger, Nidhal Selmane, and Renaud Pacalet. Silicon-level Solutions to Counteract Passive and Active Attacks. In Luca Breveglieri, Shay Gueron, Israel Koren, David Naccache, and Jean-Pierre Seifert, editors, FDTC, pages 3-17. IEEE Computer Society, 2008.

[18] Silicon Investigations. Company website: http://www. siliconinvestigations.com/.

[19] Susmit Jha and Sumit Kumar Jha. Randomization Based Probabilistic Approach to Detect Trojan Circuits. In Proceedings of the 2008 11th IEEE High Assurance Systems Engineering Symposium, HASE '08, pages 117-124, Washington, DC, USA, 2008. IEEE Computer Society.

[20] Yier Jin and Y Makris. Hardware trojan detection using path delay fingerprint. In Hardware-Oriented Security and Trust, 2008. HOST 2008. IEEE International Workshop on, pages 51-57, 2008.

[21] Marc Joye and Michael Tunstall. Fault Analysis in Cryptography. Springer LNCS, March 2011. http://joye.site88.net/FAbook.html. DOI: 10.1007/978-3-642-29656-7 ; ISBN 978-3-642-29655-0.

[22] Markus Kasper, Amir Moradi, Georg T. Becker, Oliver Mischke, Tim Güneysu, Christof Paar, and Wayne Burleson. Side channels as building blocks. J. Cryptographic Engineering, 2(3):143-159, 2012.

[23] Paul C. Kocher, Joshua Jaffe, and Benjamin Jun. Differential Power Analysis. In Proceedings of CRYPTO'99, volume 1666 of LNCS, pages 388-397. Springer-Verlag, 1999.

[24] Oliver Kömmerling and Markus G. Kuhn. Design Principles for TamperResistant Smartcard Processors. In WOST '99 (USENIX Workshop on Smartcard Technology), pages 9-20, Berkeley, CA, USA, May 10-11 1999. USENIX Association. Chicago, Illinois, USA (On-line paper). ISBN: 1-880446-34-0.

[25] Jie Li and John Lach. At-speed delay characterization for IC authentication and Trojan Horse detection. In Proceedings of the 2008 IEEE International Workshop on Hardware-Oriented Security and Trust, HST '08, pages 8-14, Washington, DC, USA, 2008. IEEE Computer Society.

[26] Bradley Hopkins Mark Beaumont and Tristan Newby. Hardware Trojans - Prevention, Detection, Countermeasures. http://www.dtic.mil/cgi-bin/ GetTRDoc?AD=ADA547668.

[27] David R. McIntyre, Francis G. Wolff, Christos A. Papachristou, and Swarup Bhunia. Dynamic evaluation of hardware trust. In Proceedings of the 2009 IEEE International Workshop on Hardware-Oriented Security and Trust, HST '09, pages 108-111, Washington, DC, USA, 2009. IEEE Computer Society.

[28] NIST/ITL/CSD. Advanced Encryption Standard (AES). FIPS PUB 197, Nov 2001. http://csrc.nist.gov/publications/fips/fips197/fips-197.pdf.

[29] Gilles Piret and Jean-Jacques Quisquater. A Differential Fault Attack Technique against SPN Structures, with Application to the AES and KHAZAD. In CHES, volume 2779 of LNCS, pages 77-88. Springer, September 2003. Cologne, Germany.

[30] SEMATECH Surface Preparation and Cleaning Conference (SPCC). Conference website: http://www.sematech.org/meetings/spcc/past.htm.

[31] Jean-Jacques Quisquater and David Samyde. ElectroMagnetic Analysis (EMA): Measures and Counter-Measures for Smard Cards. In I. Attali and T. P. Jensen, editors, Smart Card Programming and Security (Esmart 2001), volume 2140 of LNCS, pages 200-210. Springer-Verlag, September 2001. Nice, France. ISSN 0302-9743.

[32] Reza Rad, Jim Plusquellic, and Mohammad Tehranipoor. Sensitivity analysis to hardware Trojans using power supply transient signals. In
Proceedings of the 2008 IEEE International Workshop on HardwareOriented Security and Trust, HST '08, pages 3-7, Washington, DC, USA, 2008. IEEE Computer Society.

[33] Hassan Salmani, Mohammad Tehranipoor, and Jim Plusquellic. New design strategy for improving hardware Trojan detection and reducing Trojan activation time. In Hardware-Oriented Security and Trust, 2009. HOST '09. IEEE International Workshop on, pages 66-73, 2009.

[34] Martin Schobert. GNU software DEGATE. Webpage: http://www.degate. org/.

[35] Sergei Skorobogatov and Christopher Woods. Breakthrough silicon scanning discovers backdoor in military chip. In Proceedings of the 14th international conference on Cryptographic Hardware and Embedded Systems, CHES'12, pages 23-40, Berlin, Heidelberg, 2012. SpringerVerlag.

[36] Mohammad Tehranipoor and Farinaz Koushanfar. A Survey of Hardware Trojan Taxonomy and Detection. IEEE Des. Test, 27(1):10-25, January 2010.

[37] Mohammad Tehranipoor and Cliff Wang, editors. Introduction to Hardware Security and Trust. Springer, 2012. ISBN 978-1-4419-8079-3.

[38] Randy Torrance and Dick James. The State-of-the-Art in IC Reverse Engineering. In CHES, volume 5747 of $L N C S$, pages 363-381. Springer, September 6-9 2009. Lausanne, Switzerland.

[39] Randy Torrance and Dick James. The state-of-the-art in semiconductor reverse engineering. In Design Automation Conference (DAC), 2011 48th ACM/EDAC/IEEE, pages 333-338, 2011.

[40] Michael Tunstall, Debdeep Mukhopadhyay, and Subidh Ali. Differential Fault Analysis of the Advanced Encryption Standard Using a Single Fault. In Claudio Agostino Ardagna and Jianying Zhou, editors, WISTP, volume 6633 of Lecture Notes in Computer Science, pages 224-233. Springer, 2011.

[41] U.S. Department Of Defense. Defense science board task force on high performance microchip supply. http://www.acq.osd.mil/dsb/reports/ 2005-02-HPMS Report Final.pdf.

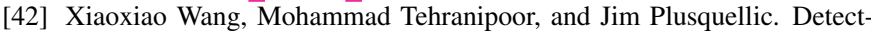
ing malicious inclusions in secure hardware: Challenges and solutions. In Proceedings of the 2008 IEEE International Workshop on HardwareOriented Security and Trust, HST '08, pages 15-19, Washington, DC, USA, 2008. IEEE Computer Society. 
ApPEndix A

Example of DFA on AES Infected Hardware Trojan Horse ON SASEBO-GII

We implemented a HTH in an AES coprocessor to facilitate Piret's DFA. The HTH gets activated when the switch is on and the first two bytes of the plaintext are equal to Oxff; it is thus an instantiation of the generic HTH depicted in Fig. 3 with parameter $N$ set to 16 . This configuration leads us to a unique key in a total of 4 encryptions. The key used in our example is same as in Appendix B of the AES standard [28]. The design is tested on a SASEBO-GII board running on the Virtex-5 FPGA.

Table IV

Cost and Performance Overhead of the Inserted HTH of Size $N=16$

\begin{tabular}{|c|c|c|c|}
\hline Design & LUT & BRAM & Frequency \\
\hline \hline HTH Free AES & 1851 & 0 & $222.477 \mathrm{MHz}$ \\
HTH Infected AES & 1861 & 0 & $218.722 \mathrm{MHz}$ \\
\hline Overhead & $0.5 \%$ & 0 & $1.6 \%$ \\
\hline
\end{tabular}

Tab IV compares the cost and performance of a HTH as described before which gets activated when the first two bytes of the plaintext are equal to $\operatorname{Oxff}$ i.e. $N=16$. The HTH is very small i.e. only 10 LUTs and causes speed degradation of $1.6 \%$ (indeed, our HTH adds one XOR gate on the critical path). This configuration helps us to easily implement Piret's Attack. Recall that the signal activation_cond[0] is an input of the trigger part of the HTH; thus both a faulted and a fault-free ciphertext can be obtained (which is required for the DFA of Piret and Quisquater). In Tab V, we give the transcript of an example of the Piret's Attack on an AES implementation with our inserted HTH.
Table V

Piret's ATTACK ON THE TROJANED AES, REVEALING THE LAST ROUND KEY

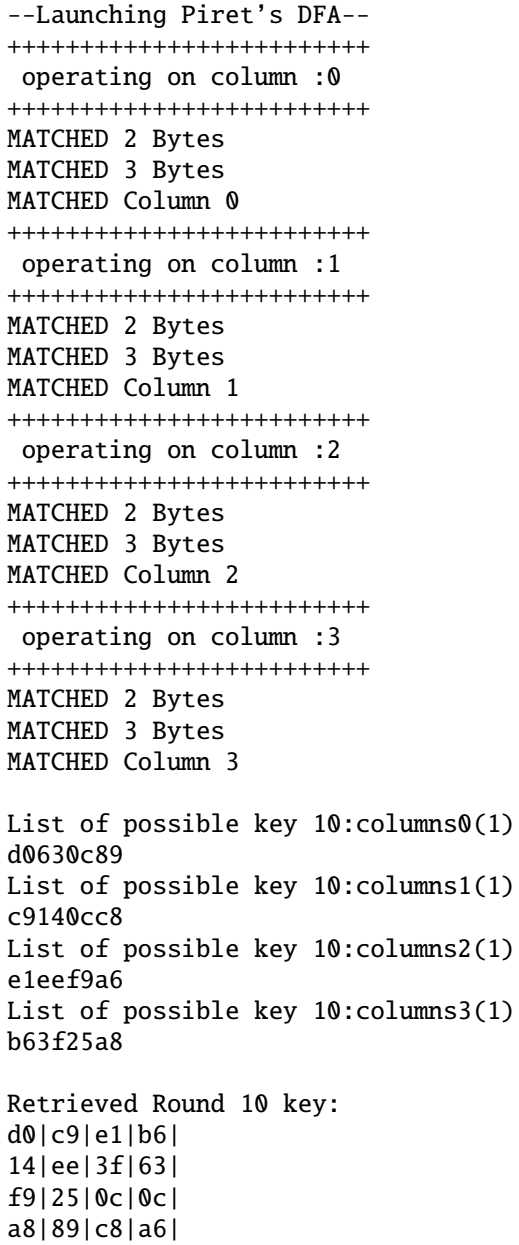




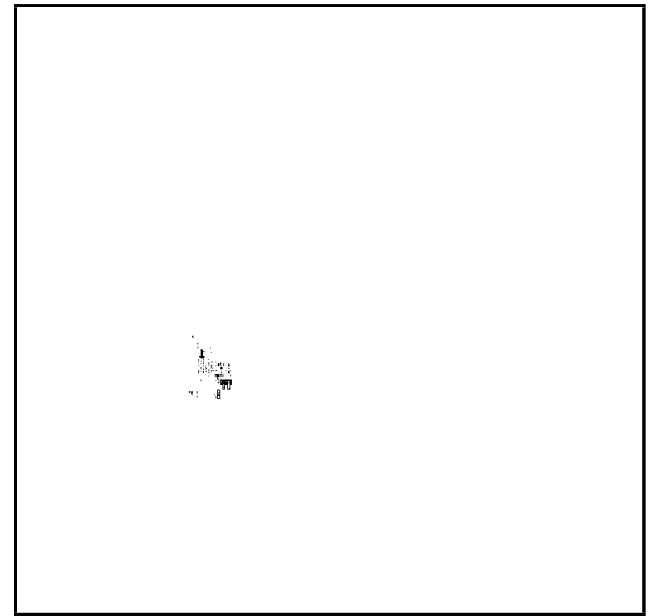

(a)

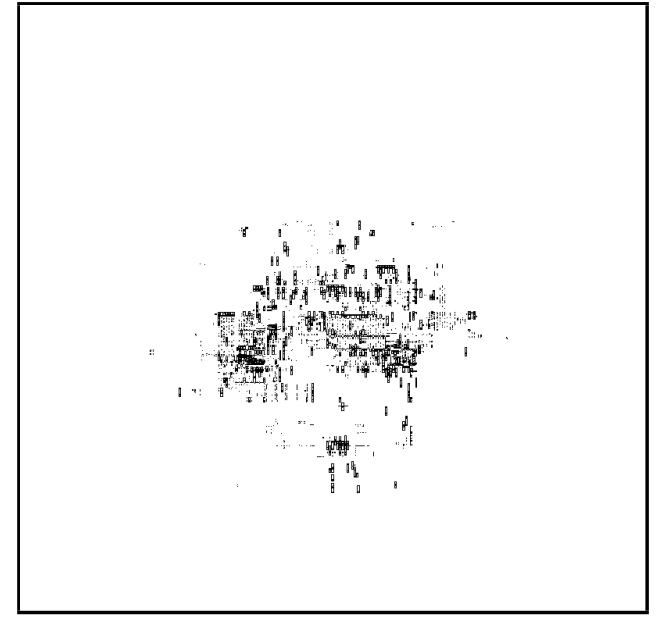

(b)

Figure 8. Pixelwise difference of AES layouts with 50\% core utilization rate for Original layout and Infected Layout with (a) 1 AND gate, (b) 128 AND gate.

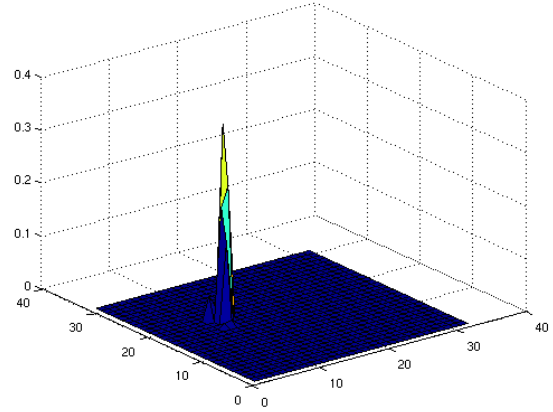

(a)

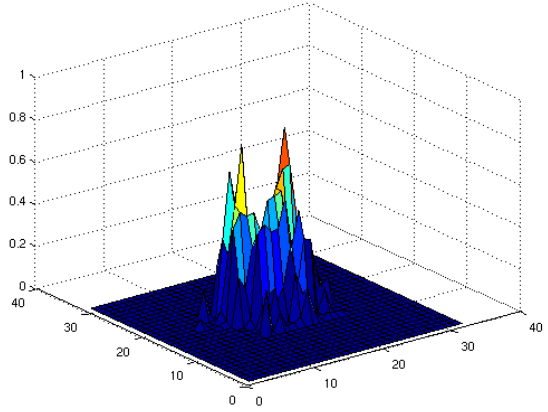

(b)

Figure 9. Grid-wise RNCC of AES layouts for with 50\% core utilization rate for Original layout and Infected Layout with (a) 1 AND gate, (b) 128 AND gate.

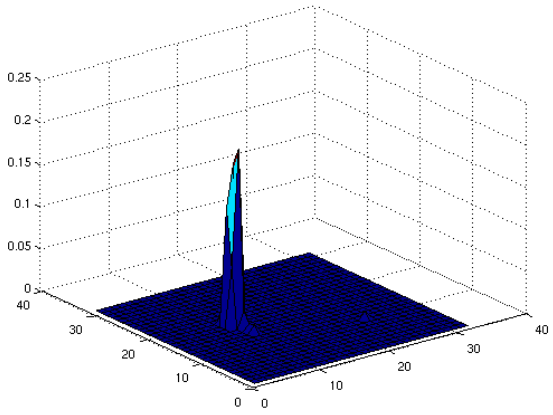

(a)

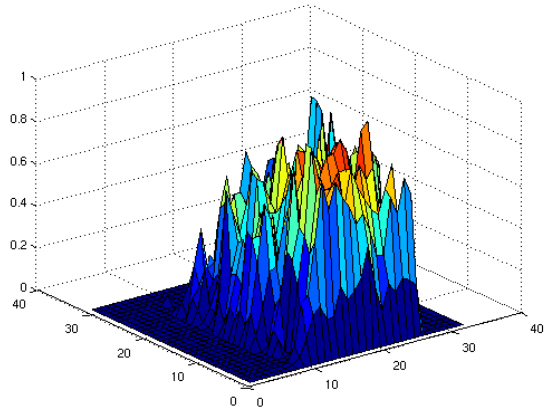

(b)

Figure 10. Grid-wise RNCC of AES layouts for with 95\% core utilization rate for Original layout and Infected Layout with (a) 1 AND gate, (b) 128 AND gate. 
Trojaned GDSII

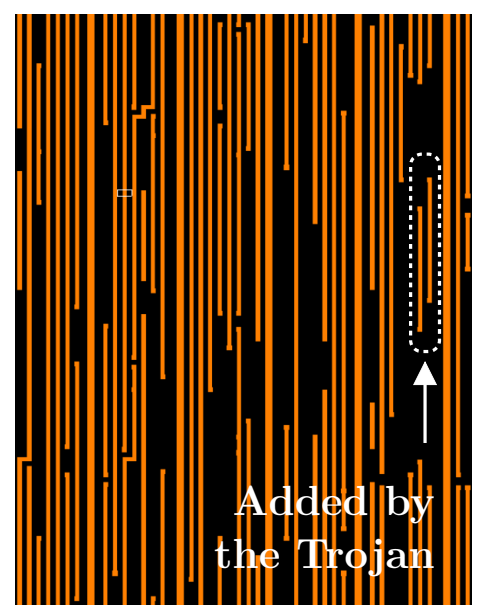

Picture

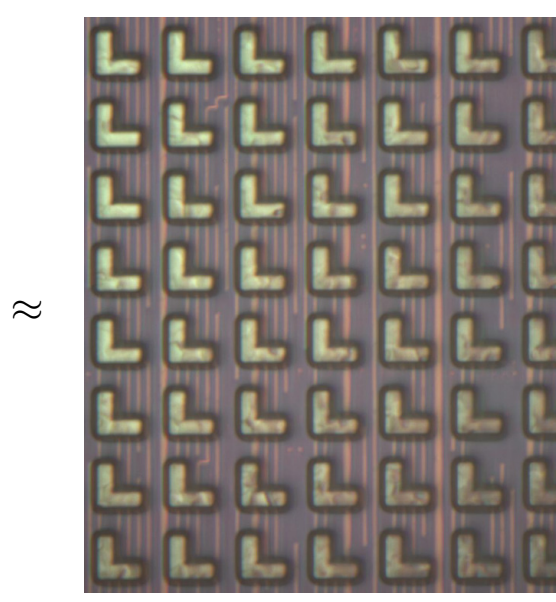

Genuine GDSII

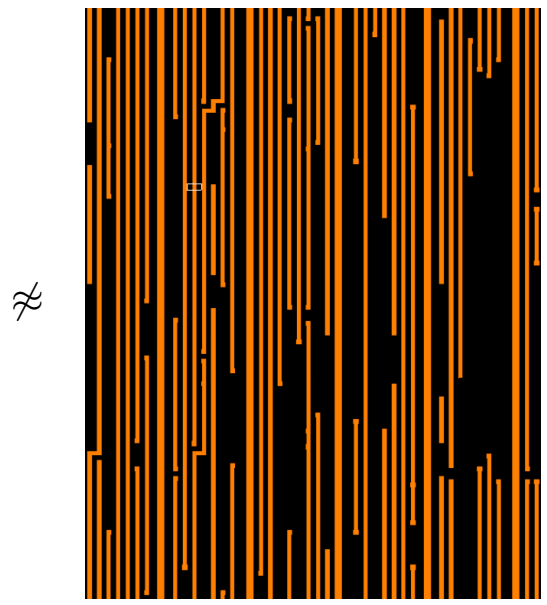

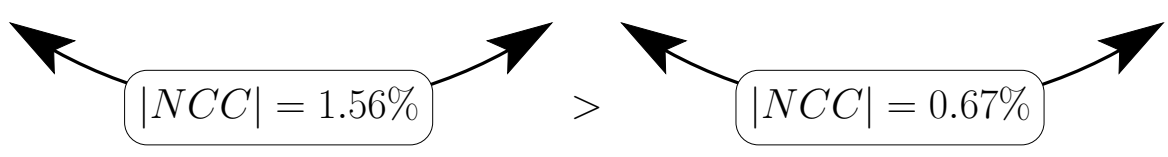

Figure 11. Cross-correlation based comparison between trojaned (lefthand side) / genuine (righthand side) GDSII and an actual picture, a microscope image of an AES area where the inserted HTH shows up (center). The designer checking for the presence of a purported HTH only knows the genuine GDSII and the picture. 